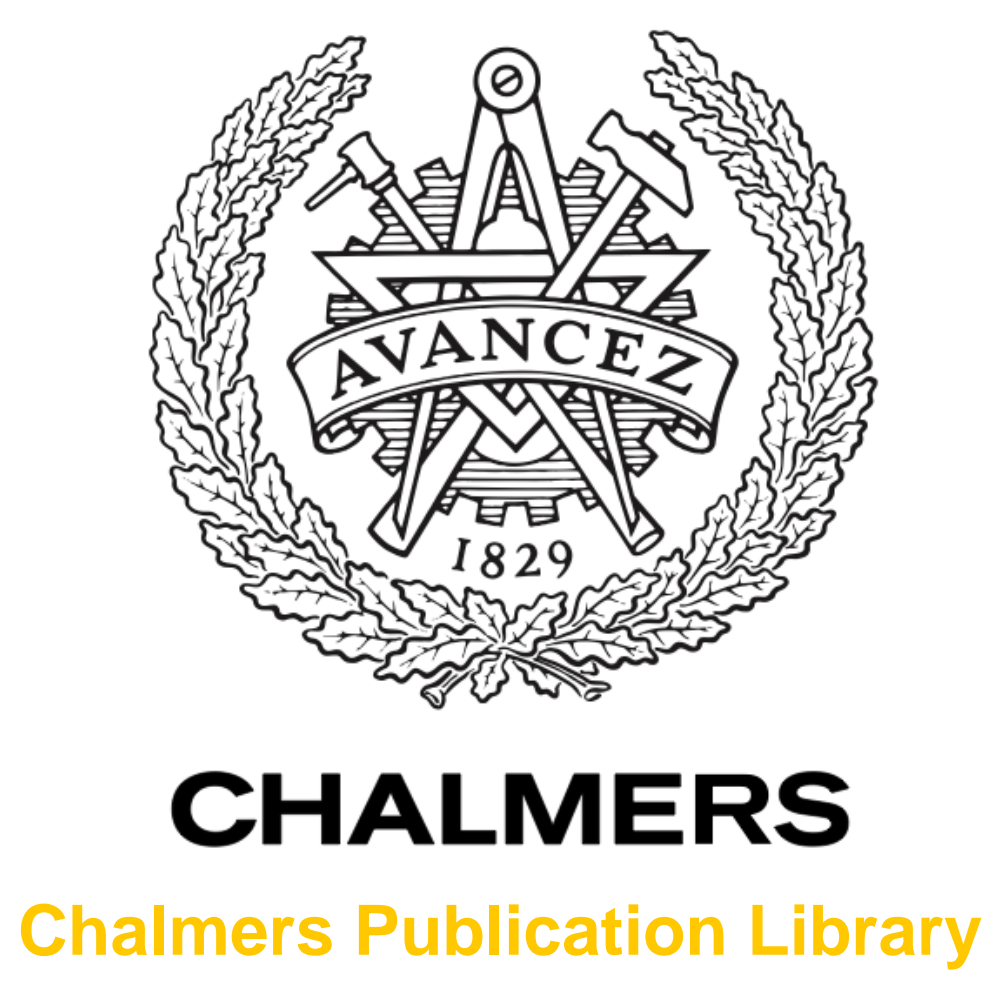

\title{
On the spectral efficiency limits of crosstalk-limited homogeneous single-mode multi- core fiber systems
}

This document has been downloaded from Chalmers Publication Library (CPL). It is the author's version of a work that was accepted for publication in:

\section{Optics InfoBase Conference Papers}

Citation for the published paper:

Luís, R. ; Puttnam, B. ; Rademacher, G. et al. (2017) "On the spectral efficiency limits of crosstalk-limited homogeneous single-mode multi-core fiber systems". Optics InfoBase Conference Papers, vol. Part F53-Networks 2017 pp. NeTu2B.2.

$\frac{\text { http://dx.doi.org/10.1364/NETWORKS.2017.NeTu }}{2 \mathrm{~B} .2}$

Downloaded from: http://publications.lib.chalmers.se/publication/251579

Notice: Changes introduced as a result of publishing processes such as copy-editing and formatting may not be reflected in this document. For a definitive version of this work, please refer to the published source. Please note that access to the published version might require a subscription. 


\title{
On the Spectral Efficiency Limits of Crosstalk-Limited Homogeneous Single-Mode Multi-Core Fiber Systems
}

\author{
R. S. Luís ${ }^{1}$, B. J. Puttnam ${ }^{1}$, G. Rademacher ${ }^{1}$, W. Klaus ${ }^{1}$, E. Agrell ${ }^{2}$, Y. Awaji ${ }^{1}$, N. Wada ${ }^{1}$ \\ ${ }^{I}$ National Institute of Information and Comm. Technology, 4-2-1, Nukui-Kitamachi, Koganei, Tokyo 184-8795, Japan \\ ${ }^{2}$ Dept. of Signals and Systems, Chalmers University of Technology, SE-412 96 Gothenburg, Sweden \\ rluis@nict.go.jp
}

\begin{abstract}
We provide a simple evaluation of the spectral efficiency limits and optimum core count of homogeneous single-mode multi-core fiber links limited by crosstalk, providing useful insight on the design requirements of these systems.
\end{abstract}

OCIS codes: (060.2400) Fiber properties; (060.2330) Fiber optics communications.

\section{Introduction}

Space division multiplexing (SDM) systems using weakly coupled core multi-core fibers (MCF) have been extensively proposed to achieve ultra high-capacity transmission (e.g. [1-3]). In particular, homogeneous single mode MCFs have been used to reach $\mathrm{Pb} / \mathrm{s}$ capacity through the use of advanced SDM techniques, such as core-coding or multi-core modulation formats and wideband wavelength division multiplexed transmission $[1,3]$. The performance of such systems may be limited by inter-core crosstalk [4]. Hence, it is imperative to investigate their spectral efficiency (SE) limits. In particular, the impact of crosstalk on the SE is related to the fiber geometry and the transmission distance. Understanding these limits may provide useful insights for the design of MCFs as well as multi-core components for SDM systems.

We present a simple theoretical evaluation of the SE limits of SDM systems limited by crosstalk in homogeneous MCFs. We show that an optimum core count may be calculated for a given transmission distance and fixed cladding diameter, assuming optimal core distribution. We apply our approach to compute the optimum core counts for MCF link distances from short-reach up to long haul assuming a set of characteristics of MCFs used in previous experimental demonstrations.

\section{Spectral Efficiency Limits in Multi-Core Fiber Systems}

In this work, we assume that transmission is supported by coherent detection and the transmitted signals have no dominant carrier. Furthermore, it will be assumed that the symbol rates are sufficiently high that crosstalk fluctuations may be ignored [5]. In these conditions, we may assume that the crosstalk follows a Gaussian distribution within each quadrature of each polarization. In addition, we will assume that the crosstalk spectrum is flat. Under these conditions

a)

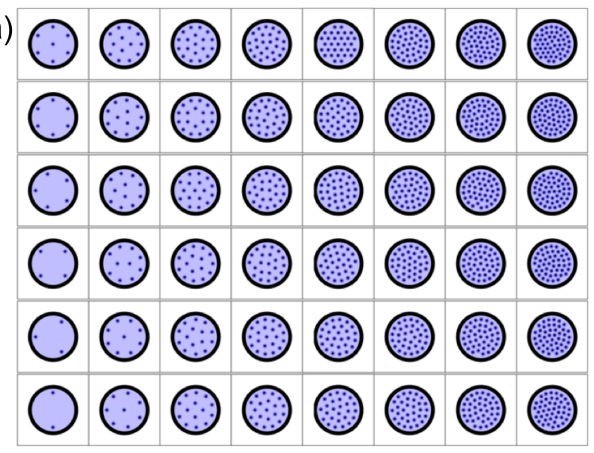

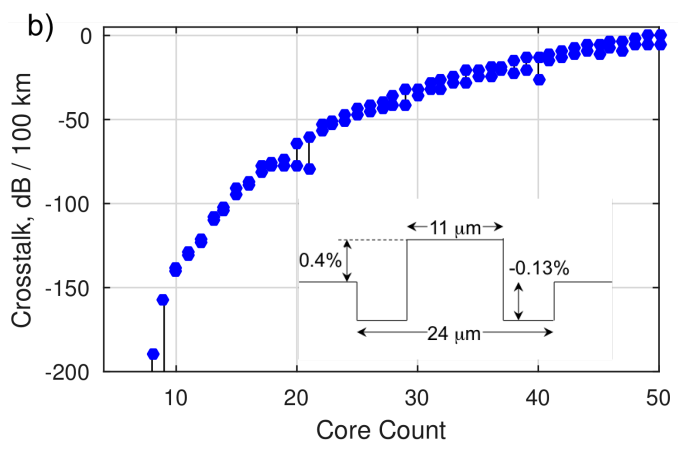

Fig. 1. a) Core arrangements maximizing core pitch for MCFs with 2 to 50 cores (a) and the corresponding estimated maximum and minimum crosstalk (b). 


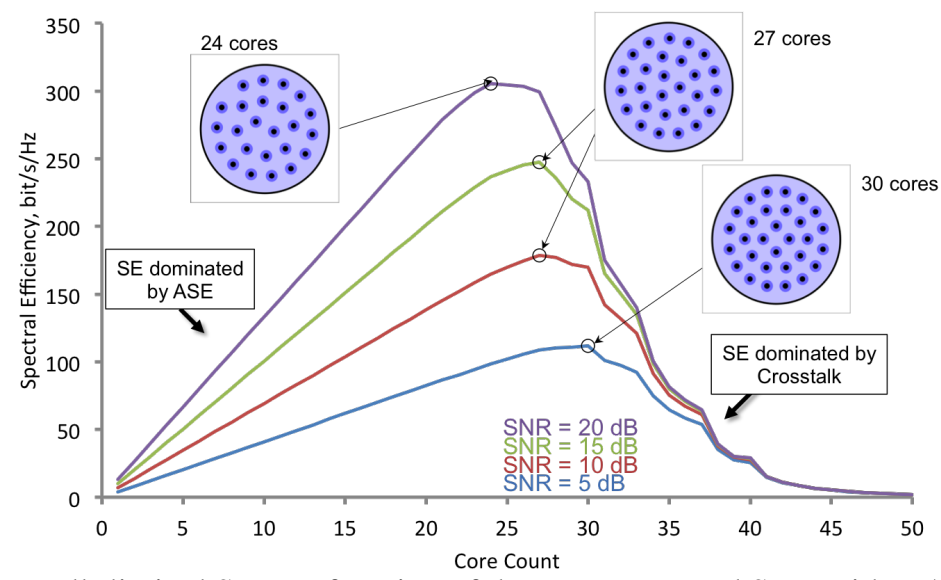

Fig. 2. Crosstalk-limited SE as a function of the core count and SNR with a $50 \mathrm{~km} \mathrm{MCF.}$

and neglecting the impact of fiber nonlinearities, the SE of the $n$-th core within an MCF transmission system is approximated by:

$$
\mathrm{SE}_{n} \approx \log _{2}\left[1+\left(\mathrm{SNR}_{n}^{-1}+\sum_{m \neq n} x_{m, n} \frac{P_{m}}{P_{n}}\right)^{-1}\right]
$$

where $\mathrm{SNR}_{m}$ is the signal-to-noise (SNR) ratio of the signal from the $n$-th core in the absence of crosstalk, $x_{m, n}$ is the crosstalk originating from core $m$ and affecting core $n$ and $P_{n}$ is the average power of the signal on the $n$-th core. Assuming uniform signal power and signal-to-noise ratio across cores yields:

$$
\mathrm{SE}_{n} \approx \log _{2}\left[1+\left(\mathrm{SNR}^{-1}+\mathrm{X}_{n}\right)^{-1}\right]
$$

with $\mathrm{X}_{n}=\sum_{m \neq n} x_{m, n}$ as the total crosstalk affecting core $n$ and $\mathrm{SNR}=\mathrm{SNR}_{n}$ as the SNR of each core. The SE of our system is given by the sum of the SEs of the different cores as $\mathrm{SE}=\sum_{n} \mathrm{SE}_{n}$. Note that we cannot assume that the $\mathrm{SE}$ is uniform across cores since the total crosstalk differs between cores. With this approximation, we can compute the crosstalk dependency on the fiber geometry. In a first order approximation assuming a fixed cladding diameter and core structure, the crosstalk between cores is mainly conditioned by the core pitch. As such, the arrangement of cores within the fiber profile may follow well known optimal arrangements of circles within circles [6]. This approach has been taken in [7] for the design of long-distance transmission MCFs. Fig. 1 shows the optimal core arrangements assuming optimization of the core pitch for MCFs with 2 to 50 cores with a fixed cladding diameter. It must be noted that the ideal arrangement of cores is often unrealizable in practice due to restrictions of the manufacturing process. Nevertheless, it provides a lower bound to the achievable crosstalk in such fibers within our assumption of dominant core-pitch.

Using the core arrangements presented in Fig. 1-a) it is possible to estimate the crosstalk in the corresponding fibers, as shown in Fig. 1-b). For this estimate, we used the model proposed in [8]. The considered core structure is shown as an inset in Fig. 1-b) and corresponds to the structure of the 22-core fiber used in multiple transmission experiments, including the current transmission throughput record using a MCF of $2.15 \mathrm{~Pb} / \mathrm{s}$ [1]. We assumed a trench-assisted core design with core, trench and cladding diameters of $11 \mu \mathrm{m}, 24 \mu \mathrm{m}$ and $260 \mu \mathrm{m}$, respectively, and a trench and core index delta $\Delta_{\text {core }}=0.4 \%$, and $\Delta_{\text {trench }}=-0.13 \%$. The outer cladding diameter was set to $33 \mu \mathrm{m}$. In this context, we may apply the channel model in (2) to estimate the SE as a function of the number of cores for a given transmission distance. An example is shown in Fig. 2 for a $50 \mathrm{~km}$ fiber. Here we have assumed SNR values from $5 \mathrm{~dB}$, up to $20 \mathrm{~dB}$. In all cases, the SE increases directly with the number of cores, as would be expected in an SDM system [9] up to a given number of cores. At that point, the crosstalk component in (2) becomes dominant, degrading the SE faster than the increase due to the number of cores. We note that the SE values shown in Fig. 2 assume the transmission of a SSC occupying all available cores. However, the $\mathrm{SE}$ of each core is strongly dependent on its position with respect to the other cores.

From Fig. 2, it is clear that for a given SNR there is an optimum number of cores, which may be estimated as a function of the SNR for a set of interesting transmission distances. Fig. 3-a) and -b) show the optimum number of cores and the SE, respectively, as a function of the SNR for 4 classes of transmission distances: $5 \mathrm{~km}$ for short reach networks, $50 \mathrm{~km}$ for metro or access networks, $500 \mathrm{~km}$ for regional networks and $5000 \mathrm{~km}$ for long-distance networks. 

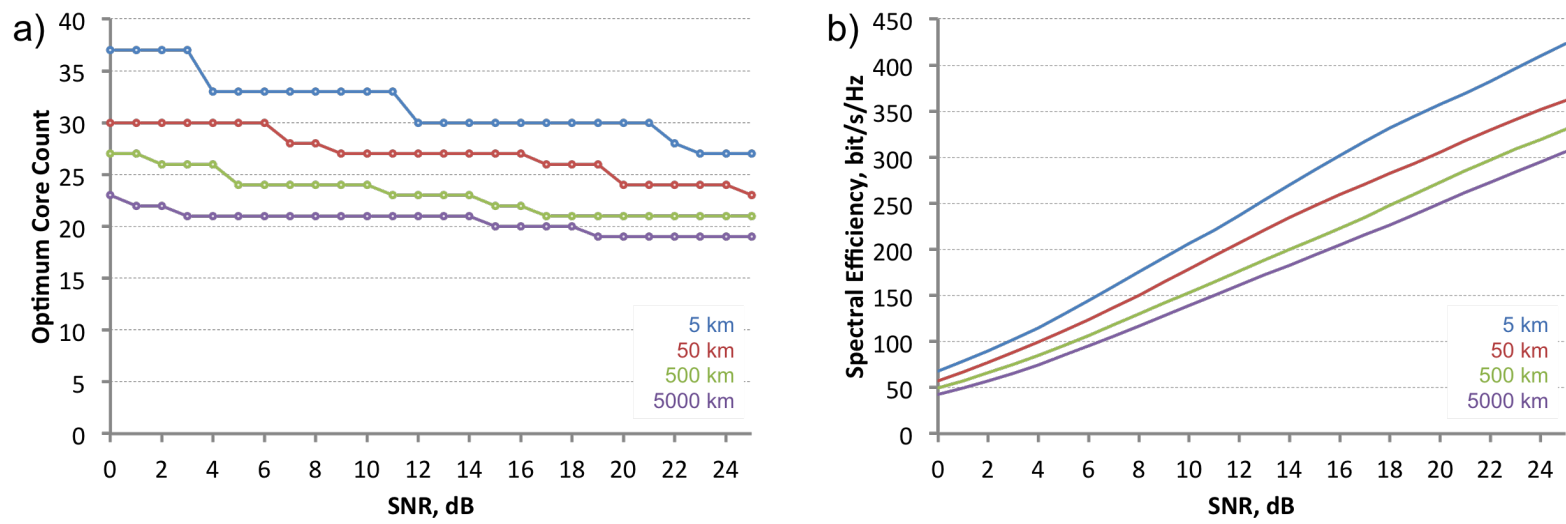

Fig. 3. Optimum core count (a) and corresponding SE (b) as a function of SNR for various reaches.

It is shown that the optimum number of cores decreases with SNR. For an arbitrarily high SNR, the optimum number of cores would converge to 1 as it would provide arbitrarily high SE. For long distance networks, the optimum number of cores changes relatively slowly within the considered SNR range. We can assume that fibers with 19 to 22 cores would be sufficient to cover a wide range of SNR values achievable in these systems. Regional networks would be able to support fibers with 21 up to 26 cores and this range would shift to 23 to 30 cores for metropolitan networks. For short-reach networks up to 37 cores could be used. Nevertheless, we must stress that our analysis is valid only for coherent transmission systems with carrier-less signals and therefore not necessarily applicable to current short-reach networks. Similar considerations may also apply to access networks.

As shown, the crosstalk-imposed SE limits of single-mode homogeneous MCF systems can be significant, limiting the practical number of cores in such fibers. However, this work disregards crosstalk-management techniques, such as direction interleaving [10] or the use of MIMO systems, which may be used to decrease the crosstalk impact and consequently improve the SE limits. Furthermore, this work disregards the use of ultra-low crosstalk heterogeneous MCFs, such as proposed in [11], which may potentially achieve substantially higher SEs. Nonetheless, our approach provides significant insight on the achievable SE limits of SDM systems using MCFs.

\section{Conclusions}

This work has presented a simple yet useful analysis of the spectral efficiency limits of spatial division multiplexing systems using homogeneous single-mode multi-core fibers. We present an evaluation of the spectral efficiency limits of such systems as a function of the number of cores in the multi-core fiber, taking into account optimized fiber geometry. Finally, we provided values for the optimum number of cores in such fibers for various transmission distances of practical interest.

\section{References}

1. B. J. Puttnam et al., "2.15 Pb/s transmission using a 22 core homogeneous single-mode multi-core fiber and wideband optical comb," ECOC, PDP.3.1 (2015).

2. T. Mizuno et al., "Dense space division multiplexed transmission over multicore and multimode fiber for long-haul transport systems," J. Light. Technol. 34(6), 1484 (2016).

3. A. Turukhin et al., "Demonstration of $0.52 \mathrm{~Pb} / \mathrm{s}$ potential transmission capacity over $8,830 \mathrm{~km}$ using multicore fiber," ECOC, P.211 (2016).

4. T. Hayashi et al., "Behavior of inter-core crosstalk as a noise and its effect on Q-factor in multi-core fiber," IEICE Trans. Commun., 97(5), 936-944, 2014.

5. G. Rademacher et al., "Modulation format-dependence of crosstalk fluctuations in homogeneous multi-core fibers," CLEO Europe, in press (2017).

6. E. Specht, "Packings of equal and unequal circles in fixed-sized containers with maximum packing density," http://www.packomania.com.

7. T. Nakanishi et al., "Spatial-spectral-efficiency-enhanced multi-core fiber," OFC, Th3C.3 (2015).

8. F. Ye et at., "Simple analytical expression for crosstalk estimation in homogeneous trench-assisted multi-core fibers," Opt. Express 22(19), 23007 (2014).

9. R. Essiambre, and R. Tkach, "Capacity trends and limits of optical communication networks," Proc. of the IEEE 100(5), 1035 (2012).

10. A. Sano et al., "Crosstalk-managed high capacity long haul multicore fiber transmission with propagation-direction interleaving," J. Lightwave Technol., 32(16), 2771 (2014).

11. K Takenaga et al., "High-density multicore fibers," OFC, W1F.1 (2016). 